NUCLEAR INSTRUMENTS AND METHODS 19 (1974) 5I-60; C) NORTH-HOLLAND PUBLISHING CO.

\title{
THE UNIVERSITY OF MICHIGAN NEUTRON TIME-OF-FLIGHT FACILTTY*
}

\author{
W. C. PARKINSON, J. F. PETERSEN, R. H. DAY, R. M. POLICHAR, P. F. JULIEN and D. C. DUPLANTIS
}

Cyclotron Laboratory, Department of Physics, The University of Michigan, Ann Arbor, Michigan 48105, U.S.A.

Received 5 March 1974

The details of the neutron time-of-flight instrumentation asscciated with The University of Michigan $83^{*}$ cyclotron are described and examples of capabilities of the instrument are given.

\section{Introduction}

The technique of ineasurement of neutron energies by time of flight has been in use for many years ${ }^{1}$ ). As a result of the advances in accelerator technology and fast electronics, the precision of measurement has continued to improve. The state of the art is now such that useful nuclear spectroscopic information can be extracted for even the heaviest nuclei using a variety of charged-particle induced reactions at energies sufficient to overcome the Coulomb barrier. As a single example, it is now possible to investigate the vary interesting two-proton transfer reaction $\left({ }^{3} \mathrm{He}, n\right)$ in the heaviest elements at ${ }^{3} \mathrm{He}$ energies above the Coulomb barrier in spite of the usually large positive $Q$ values and the very small cross sections. Further, the modern instrumentation permits rather precise measurements of total and partial neutron cross sections as a function of energy at energies up to man, $\mathrm{MeV}$.

The time-of-flight spectrometer now in use sith The University of Michigan's $83^{\prime \prime}$ cyclotron facility is an extension and modification of earlier versions ${ }^{2.3}$ ) the basis of which is the natural phase bunching of the circulating beam in the cyclotron. The purpose of this paper is to describe the facility and to indicate the type and quality of measurements that are being made.

\section{Description of the facility}

The floor plan is shown in fig. 1 . The beam circulating in the cyclotron is gated at the ion source so that narrow pulses of beam at any sub-multiple of the cyclotron if frequency can be extracted from the cyclotron and brought to an intermediate focus at $F 1$ by means of a pair of $4^{\prime \prime}$ quadrupole magnets Q1. After passing through a stripping foil (optional), a dispersive element M1, a second focus $F 2$, energy defining slits $S 3$, quadrupole doublet $Q 2$ and a switching magnet $M 2$, the beam pulses enter the uniform field "target"

- Work supported in part by the U.S. Atomic Energy Commission. magnet M3. The target, mounied in the gap of M3, can be moved in azimuth through the gap to vary the neutron scattering angle. The quadrupole Q2 in conjunction with the switching magnet M2 provides a parallel beam onto the target. Neutrons produced in the target and emitted in the direction of the neutron tunnel are collimated by neutron slits NS1 and NS2 and impinge on an array of neutron detectors (D) placed at the end of the tunnel. The array has a sensitive area of $0.405 \mathrm{~m}^{2}$, so that for 1 flight path of $\mathbf{4 0} \mathrm{m}$ the array subtends a solid angle of $2.53 \times 10^{-4} \mathrm{sr}$. Because the extent in azimuth of the present target magnet is only $90^{\circ}$,

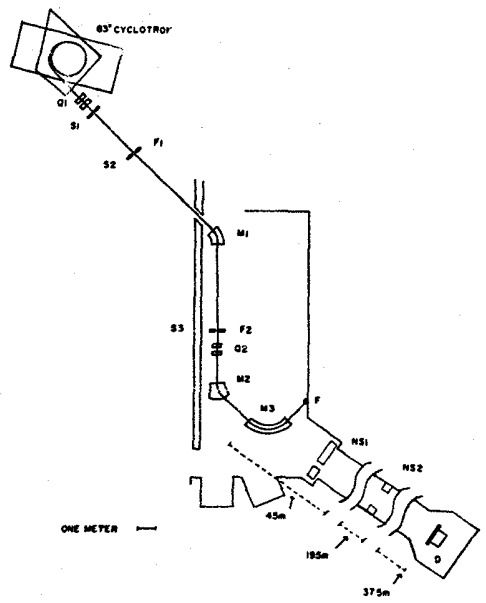

Fig. 1. The floor plan of the time-of-flight facility. Targets are moved in azimuth through magnet $M 3$ to vary the scattering angle. 
measurements of angular distributions are limited in angle from $-10^{\circ}$ to $+80^{\circ}$.

The particle current through the target is measured by means of a Faraday cup (F) located at the beam dump. If the multiple scattering of the incident particles by the target is relatively large, as is the case for heavy ions or thick targets, the Faraday cup is mounted a fixed distance from the target and moved in azimutin with it. In this case the cup must be shielded from the detectors. (A disadvantage of this method of varying the scattering angle is that the magnet aperture sets a limit on the acceptable nultiple scattering angle and kence target thickness.)

When singly charged light ioris such as ${ }^{3} \mathrm{He}^{+}$(or heavy ions) are accelerated in the cyclotron, the remaining electron is stripped in the target. As a result of the decrease in magnetic rigidity, the ions are not collected at the Faraday cup but rather deflect into the walls of the vacuum chamber. To avoid this a stripper foil is placed at $F 1$ and the ion optics adjusted to bring the fully stripped ions onto the target.

In addition to reaction studies such as $(\mathrm{d}, \mathrm{n})$ and $\left({ }^{3} \mathrm{He}, \mathrm{n}\right)$, the present system is particuiarly adaptable to measurements of total and partial neutron cross sections in the energy range 5-50 MeV. The energy of the monoenergetic neutrons produced by the $D(d, n)^{3} \mathrm{He}$ or $T(d, n)^{4}$ He reactions can be varied continuously by varying the scattering angle, and/or by varying the deuteron energy.

At present the overall time resolution is limited primarily by the time spread of the beam pulses from the cyclotron. Phase widths of the order of $3^{\circ}$ fwhm of the of cycle can be obtained routinely, which for if frequencies of the order of $7 \mathrm{MHz}$ corresponds to a time spread of about $1.2 \mathrm{~ns}$. This can be reduced in the external ion-optical system but only at the expense of beam intensity. Taken in quadrature with the time resolution of the present detector system, the result is a resolution, neglecting target effects, of approximately $1.5 \mathrm{~ns}$. Thus the energy resolution at $40 \mathrm{~m}$ flight path is $9 \mathrm{keV}$ for $5 \mathrm{MeV}$ neutrons and $170 \mathrm{keV}$ for $30 \mathrm{MeV}$ neutrons. Methods of improving the resolution are discussed below.

The key elements in the system are the gated ion source, the quality of the circulatirig beam in the cyclotron, the external ion-optical system, the detector array and the timing system. The details of these are discussed in the rollowing sections.

\section{The gated beam}

The gated ion source, in routine use for neutron time-of-flight and a variety of other types of measure- ments, has been described in detail previously ${ }^{4}$ ). The principal features are that ions of any charge state can be gated with $100 \%$ efficiency, the current extracted from the plasma is space-charge limited (Childs' Law) and the current injected into the dee is independent of dee voltage. These features lead to some interesting consequences.

The gated source utilizes a tetrode geometry. In analogy with the tetrode vacuum tube, it consists of the conventional non-gated ion source (cathode) and puller (plate) with two plane-parallel electrodes, the control electrode (grid) and the shield electrode (screen grid) interposed between them. The control and shield electrodes are formed from tantalum sheet $\mathbf{0 . 0 1 0 ^ { \prime \prime }}$ thick and each contains a single slit. The dimensions of the slits are varied depending on the ions being accelerateó. For singly ionized light ions nominal dirnensions are $0.5^{\prime \prime}$ high and $0.020^{\prime \prime}, 0.020^{\prime \prime}, 0.040^{\prime \prime}$, and $0.020^{\prime \prime}$ wide for the cathode, control electrode, shield electrode, and puller, respectively. For doubly ionized ions, such as ${ }^{3} \mathrm{He}^{++}$and heavy ions, typical dimensions are $0.5^{\prime \prime}$ high and $0.040^{\prime \prime}, 0.020^{\prime \prime}, 0.040^{\prime \prime}$, and $0.020^{\prime \prime}$ wide. Nominal interelectrode distances are $0.025^{\prime \prime}$ cathode to control electrode, $0.031^{\prime \prime}$ control to shield electrodes, and $0.312^{\prime \prime}$ shield to puller. The slits are aligned on the central trajectory of the ions extracted from the plasma.

The function of the shield electrode is to shield the cathode from the electric field of the puller. For dc operation the contiol and shield electrode potentials are typically $-2 \mathrm{kV}$ and $-500 \mathrm{~V}$, respectively. In the gated mode a negative gating voltage, synchronized with and at any sub-multiple of the if frequency, is applied to the control electrode and a constant and negative dc voltage is applied to the shield. The gating voltage is manufactured by picking an if reference signal from the dee, passing it through a variable time delay, and counting down to the desired mode $(f / 2, f / 3, \ldots)$ with standard $T T^{*}$. circuitry. The resulting square wave is clipped, amplified, and applied to the control grid through a transmission line incorporated on the ion source tube. The gating pulse is approximately $3 \mathrm{kV}$ in amplitude with 24 ns rise and fall times and a 30 ns flat top.

The fact that the current injected into the dee is independent of dee voltage permits the selection of a narrow phase group at any if phase for acceleration. This is a useful feature in obtaining high-quality extracted beams ${ }^{5}$ ).

For most ion sources the beam current is spacecharge limited and for plane-parallel geometry varies as $V^{2} / d^{2}$ (Childs' Law), where $V$ is the cathode-pulier 
potential difference and $d$ their separation. For the gated source $\boldsymbol{V}$ is the control electrode-cathode potential difference and $d$ the separation. Thus the yield from the gated source with $V=3 \mathrm{kV}$ and $d=0.025^{\prime \prime}$ is a factor of two greater than for the normal source with $V=50 \mathrm{kV}$ and $d=0.310^{\prime \prime}$. In general, ions of other species exist in the plasma (with deuterium for example the plasma will contain the molecular ions $D_{2}^{+}$and $D_{3}^{+}$as well as $D_{1}^{+}$). It can be shown ${ }^{4}$ ) that the current density of any one species will be

$$
J_{1}=\frac{4}{9} \varepsilon_{0} \frac{\left(2 e_{1} / m_{1}\right)^{\frac{1}{2}}}{\left(1+\sum_{i=2}^{k} \frac{n_{i} e_{i}}{n_{1} e_{1}}\right)} \frac{V^{\frac{3}{2}}}{d^{2}},
$$

where $n_{i}$ and $e_{i}$ are the number per $\mathrm{cm}^{3}$ and the charge of the $i$ th species, respectively. This follows from the fact that $\mathrm{div} j_{i}+\partial \rho_{i} / \hat{\partial} t=0$ for each ion species. For the hooded type ion source $)^{6}$, the ratio of $\left(n_{2} e_{2} / n_{1} e_{1}\right)$ for $\left({ }^{3} \mathrm{He}^{++} /{ }^{3} \mathrm{He}^{+}\right)$is of the order of 1:50. As a result, the ${ }^{3} \mathrm{He}^{++}$current density is relatively small. It is for this reason that ${ }^{3} \mathrm{He}^{+}$is accelerated, extracted and then stripped to ${ }^{3} \mathrm{He}^{++}$for use with the time-of-flight spectrometer. For deuterium the ratios ${ }^{6}$ ) of $\mathrm{D}_{1}^{+}, \mathrm{D}_{2}^{+}$ and $D_{3}^{+}$are 1:2:i.5. As a result the yield of $D_{1}^{+}$is about a factor of 5 less than might naively be expected. The unwanted species $D_{3}^{+}$which would be accelerated on the third sub-harmonic of the if is eliminated after extraction from the ion source by a baffle mounted behind the puller. (The $D_{2}^{+}$will only be accelerated through the first half-turn.)

The use of a gated ion source in time-of-flight measurements puts stringent requirements on the geometry and stability of the internal orbits within the cyclotron. This problem has been investigated in detail and is discussed in a separate paper ${ }^{5}$ ). It need only be noted here that even though the gating of the ion source is $100 \%$ effective, the external beam will be gated $100 \%$ only if single-turn extraction from the cyclotron is achieved.

\section{The external ion-optical system}

The ion-optical system connecting the cyclotron and the target magnet was designed to accommodate a variety of measurements in addition to neutron spectroscopy by time-of-fight. It is a low-resolution system with $E / \Delta E=1000$ for a source width of $1 \mathrm{~mm}$. Because the target magnet is sone $23 \mathrm{~m}$ from the exit of the cyclotron, it was import int to insure not only that the ion-optical system could provide to the target a nearly parallel beam of const int and small cross-sectional area as the target was roved in azimuth through the target
TABLE 1

Properties of three uniform-field dipole magnets.

\begin{tabular}{lrlrrr}
\hline Magnet & $\begin{array}{c}\text { Bend } \\
\text { angle }\end{array}$ & $\begin{array}{c}\text { Radius } \\
\text { of curva- } \\
\text { ture (m) }\end{array}$ & $\begin{array}{c}\text { Field } \\
\text { index } n\end{array}$ & $\begin{array}{c}\text { Entrance } \\
\text { angle }\end{array}$ & $\begin{array}{c}\text { Exit } \\
\text { angle }\end{array}$ \\
\hline M1-Analyzer & $45^{\circ}$ & 0.0 & 0.0 & $+12.7^{\circ}$ & $+12.7^{\circ}$ \\
M2-Switching & $0-45^{\circ}$ & $1.0(\min )$ & 0.0 & 0.0 & $0-22.5^{\circ}$ \\
M3-Target & $90^{\circ}$ & 1.5 & 0.0 & 0.0 & 0.0 \\
\hline
\end{tabular}

magnet, but also that it did not introduce unacceptably large time spreads in the beam pulses. A computer program (a modified version of OPTIK*) was used to calculate fight times for ions following different paths through a given configuration. The elements of the configuration and the location of foci were adjusted to minimize the time spread while maintaining the resolving power and the beam size at the target position.

The properties of the three uniform-field $(n=0)$ dipole magnets (M1, M2, and M3) are listed in table 1 . Slit Sl (fig. 1) is used to limit the divergence of the beam accepted into the line and thus defines the extreme rays which determine the time spread in the beam pulse. Slit S2, at the horizontal focus of Q1, is used to define an effective source for the remaining elements. The dipole magnet $M 1$ disperses the beam, and in conjunction with the energy-defining slits $\mathbf{S} 3$ provides the required resolving power up to $E / \Delta E=1000$. The $3^{\prime \prime}$ quadrupole $\mathrm{Q} 2$ and the switching magnet $\mathrm{M} 2$ permit the beam to be focused onto the target when located at any azimuth in target magnet M3. Because the divergence through $\mathbf{M} 3$ is small, all the beam is collected by the Faraday cup regardless of the target position (with the exception of large multiple scattering noted above).

The performance of the ion-optical system was determined by measurements of the width in time of the beam pulses at the entrance to the beam line and at the target using a diagnostics probe having a time resolution of $300 \mathrm{ps}$. The measured increase was in agreement with the values calculated. For the purpose of the measurements, $11 \mathrm{MeV}$ deuterons, the lowest-velocity ion available with of acceleration on the fundamental mode, werf used. When all the beam extracted from the cyclotron was brought to the target, the increase was approximately $2 \mathrm{~ns}$. This was reduced to 400 ps by limiting the angular divergence of the beam with slits SI at the expense of a factor of four in beam intensity.

- Obtained from Lawrence Berkeley Laboratory, UCRL-9727. 
For ions of higher velocity, the time spread is reduced proportionally.

The resolving power of the ion-optical system was measured by comparing the width of the image of the cyclotron source at the energy-defining slits $S 3$ with the width of the image of the same beam sent through the high-resolution charged-particle beam line ${ }^{7}$ ). The value $E \cdot \Delta E=1000$ is in agreement with the calculated value.

\section{Targets}

The targets are mounted on a carriage designed to hold two foil targets or a gas cell. The carriage moves on a guide rail through the gap of magnet M3 to vary the scattering angle. The target holder, shown in fig. 2 , can be rotated through $90^{\circ}$ without breaking vacuum to expose either of the target frames to the beam. Generally, one frame is left empty so that background measurements can be made. The aperture is defined by a lead mask with an opening $0.75^{\prime \prime}$ high by $3^{\prime \prime}$ wide, moanted just in front of and insulated from the target. Current to the mask can be measured, and this feature is used as an aid in centering the beam on the aperture.

The gas cell, shown in fig. 3 , is $1^{\prime \prime}$ thick and $8^{\prime \prime}$ wide. The entrance aperture is again defined by a lead mask. The cell windows are generally of thin nickel, but for higher energies, such as $20 \mathrm{MeV}^{3} \mathrm{He}, 0.0003^{\prime \prime}$ tantalum is used because of the higher Coulomb barrier and low $Q$ value for the $\left({ }^{3} \mathrm{He}, \mathrm{n}\right)$ reaction. With such thick windows, the root-mean-square multiple-scattering angle is large and would result in scattered beam spraying the magnet pole tips and the aluminum duct producing a large neutron background. To avoid this, the singly ionized ${ }^{3} \mathrm{He}^{+}$, rather than being stripped at

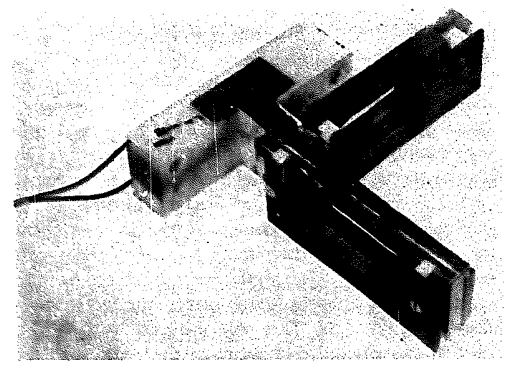

Fig. 2. Target holder. The two target frames, each with a lead mask, are mounted to the carriage via a pivot to permit either - frame to be rotated into the beam.
S2, is allowed to strip in the entrance window of the cell. The ${ }^{3} \mathrm{He}^{++}$ions then follow the trajectory indicated in fig. 3 and are collected in the lead beam stop. The beam stop travels through the magnet with the gas cell. This method works for ${ }^{3} \mathrm{He}$ energies up to about $20 \mathrm{MeV}$ which is well below the Coulomb barrier for $\mathrm{Pb}$; in addition the $Q$ value for $\left({ }^{3} \mathrm{He}, \mathrm{n}\right)$ on lead is unusually low. The integrated beam current is determined both by charge integration of the electron current to the cell and by ion current to the beam stop. The gas line to the cell is valved outside the vacuum tank to permit filling and emptying the cell at any position in the magnet for ease in taking background spectra.

\section{The detector array}

In time-of-flight measurements the uncertainty $\Delta E$

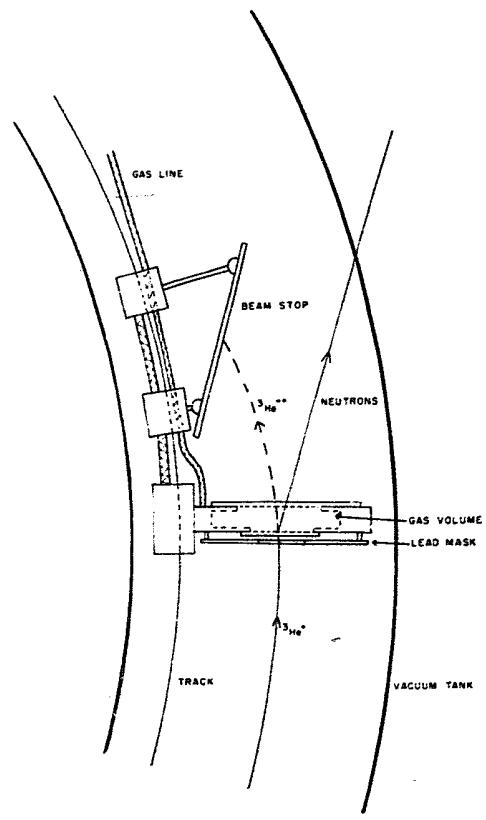

Fig. 3. The gas-cell geometry. 
in the energy $E$ of the particle is $\Delta E=2 E \Delta t / t$. A number of factors conspire to set a lower limit on $\Delta t$, and to improve the resolution it is customary to increase $t$ by increasing the flight path. This, however, reduces the solid angle subtended by the detector. Increasing the cross-sectional area of the detector to regain solid-angle results in an increase in $\Delta t$ due to the increased lightcollection time in the detector. It is easily shown that for a single detector and a given solid angle $\Omega, \Delta E \sim E^{\frac{2}{2}} \Omega^{\frac{1}{2}}$, so that the resolution is independent of the flight path.

While there are various solutions to the problem, the one we have adopted is to construct an array of detectors ${ }^{8}$ ) each of a fixed radius and therefore fixed light-collection time. By increasing the number of elements in the array, the solid angle can be increased indefinitely. The array presently in use has ten elements, as shown in fig. 4.

Each of the ten disk-shaped cylindrical containers is made of aluminum and has an active volume $8 \frac{15}{50}$ in diameter by 1 " thick. The interior is painted with NE560 white reflective epoxy and filled with NE213 liquid scintillator in a pseudocumene base solvent. The total volume of scintillator is approximately $10 \mathrm{l}$. Each

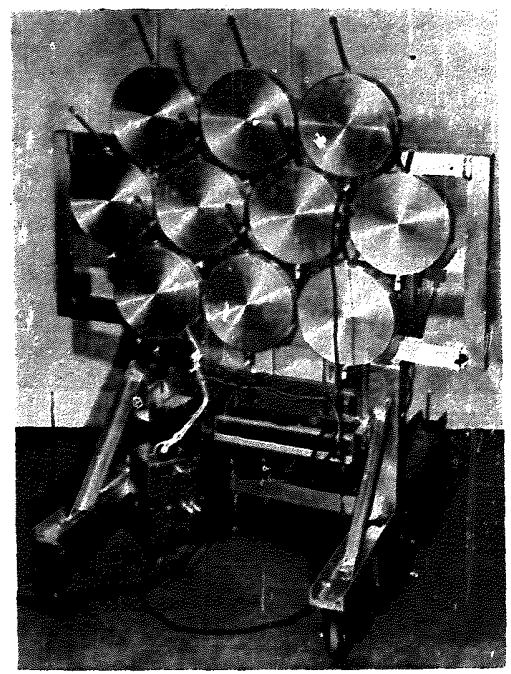

Fig. 4. The ten-element detector array. detector has its own phototube (RCA8575), mounted on the axis of the disk and immersed in the liquid, and timing discriminator (a modified Ortec 270 constantfraction timing unit). The fast logic signals from the ten discriminators are added with essentially no degradation in time resolution. The linear signals, after being added, are sent to a delay-line amplifier for energy analysis and pulse-shape discrimination. The relative gains of the photomultipliers are adjusted using a variable fan-out high-voltage supply. The tubes and timing units have been matched so that full advantage can be taken of the wide dynamic range of the constant-fraction discritninators. Time variations between tubes due to minor differences in high voltage or temperature crift are compensated to within $100 \mathrm{ps}$ by means of calibrated delay lines between each counter and the fast adder. A block diagram of the counter electronics is shown in fig. 5. A pulse-shape discrimination circuit employing conventional leading-edge-to-zero-crossing-point timing was used to eliminate pulses from $\gamma$-rays and certain cosmic rays. For large-area counters this circuit gave better results than ore employing constantfraction rise time, but at the expense of having to timematch the linear signals as well as the fast signals. The cosmic-ray background can be reduced an additional $10 \%$ using standard anticoincidence techniques but the circuitry for doing this has yet to be installed.

To facilitate rapid checking of the relative timing of the counters, a light-emitting diode (LED) has been installed in each scintillator. The light pulses from the diodes can be attenuated by small camera irises. An electrical pulse, formed by discharging a precision capacitor through a mercury relay into a $50 \Omega$ line, provides a trigger signal and drives the LEDs. The RC time constant associated with each LED is a djusted to reproduce the pulse shape from a detected particite.

The array was tested using $\gamma$-rays produced by deuterons circulating in the cyclotron striking a carbon target. By narrowing the phase width of the circulating beam, $\gamma$-ray pulses of $600 \mathrm{ps}$ fwhm, as citermined by the diagnostic beam probe, were produced. Using these "flashes", the several time constants in each fast discriminator circuit were adjusted to optimize the response. Such an adjustment is essential since the shape of the light pulse is determined by the geometry of the counter. The time resolution of the array was determined to be $(900 \pm 50) \mathrm{ps}$ and is no more tinan $50 \mathrm{ps}$ larger than that of a single detector.

Naively, the resolution might be expected to be less than 500 ps corresponding to the time for light to travel the radius of the detector. As an aid in understanding the essential parameters, the inherent resolution was 
W, C. PARKINSON et al.

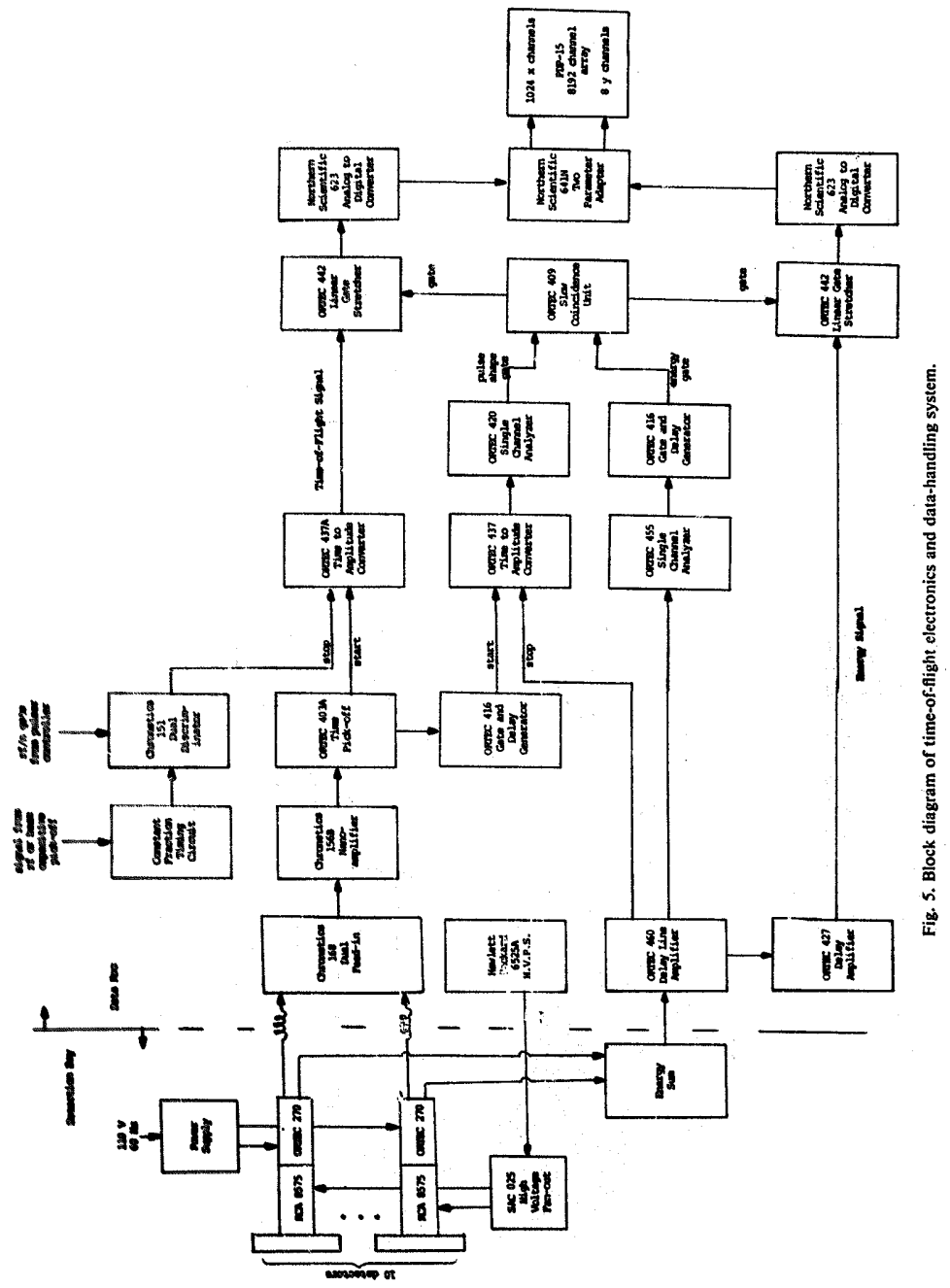




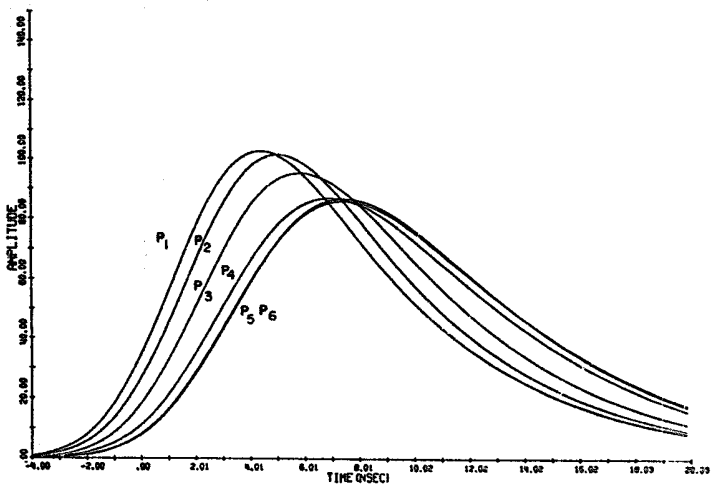

Fig. 6. Calculated phototube anode-pulse shapes vs detection point $P$ in scintillator. For points $P_{1}-P_{6}, R=0,3.3,5.2,7.1$. 9.0, åd $11.0 \mathrm{~cm}$, respectively.

calculated using a computer code written to perform a Monte Carlo calculation of the time response of the photomultiplier output to an event occurring at an arbitrary point $P$ in the liquid. In the program, a large number of photons are emitted isotropically from $P$ at the same instant. The program follows each photon as it reflects about the interior of the scintillator volume until it is either lost to the pulse or strikes the photocathode. The anode pulse resulting from the large number of photons is computed by convoluting the single-electron response of the photomultiplier. The anode pulses for six points $P_{1} \ldots P_{6}$ across the face of the detector with $P_{6}$ at a larger radius than $P_{1}$ are shown in fig. 6. The external electronics is designed to trigger at the $20 \%$ point of the pulse amplitude, and this results in a spread in triggering time between $P_{1}$ and $P_{6}$ of about $1.8 \mathrm{~ns}$. This large time spread originates from two principal sources, the finite transit time across the radius of the scintillator of the photons contributing to the leading edge of the pulse and a distortion of the pulse shape for events occurring at larger radii. When the time response is weighted according to volume, the calculated time spread for the detector is 0.9 ns, in good agreement with the measured value. This calculation, together with a calculation of the detection efficiency for neutrons, is reported separately ${ }^{9}$ ).

The detection efficiency of the array for neutrons in the energy range $0.10-20 \mathrm{MeV}$ as a function of the energy discriminator cut-off is shown in fig. 7. The calculation included single and multiple n-p scat?ering. secondary interactions such as $\left(n,{ }^{32} \mathrm{C}\right)$, and the response function of the scintillator and photolwbe for events occurring at different radii.

To date only a rough measurement of the efficiency has been made for incident neutrons of $13 \mathrm{MeV}$ asing the $D(d, n)^{3}$ He reaction. (The measurements will be refined and extended to cover the range 5-30 MeV.) The measured value, with a low energy cut-off of $1 \mathrm{MeV}$, was $(13 \pm 2.5) \%$ which is to be compared the calculated value of $13 \%$.

\section{Data handling}

The inputs to the data-handling system, showa in fig. 5; are the fast "start" signal and the energy signal, both from the detector array, and the stop signal related to the beam pulse on target. The start siswal is generated from the summed fast logic signals from the ten detectors. The sum signal is amplified and farmed out by an Ortec 403A time pick-off circuit which sap" plies the start pulse to an Ortec 437A time-to-amplitude converter (TAC). The stop signal is obtained from a capacitive pick-off described below. The output of the TAC is coupled to the input of a PDP-15 through an Ortec $\mathbf{4 4 2}$ linear gate and stretcher. The gate sigms is obtained from the $n-\gamma$ discriminator. The summed energy signal is amplified by an Ortec 460 delay-line 


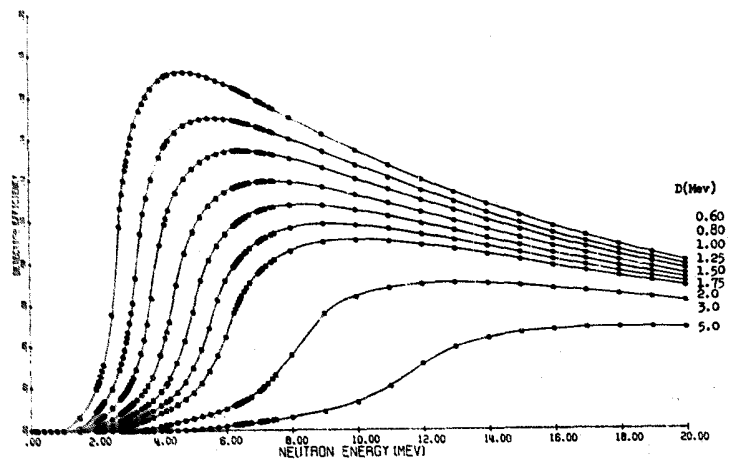

Fig. 7. Neutron detector efficiency vs neutron energy. The parameter $D$ is the low-energy discriminator cutoff.

amplitier and fed to the zero-crossing $n-\gamma$ discriminator ${ }^{(0)}$. A window is used on the energy signal bcause those cusmic-ray events which saturate the energy channel produce a neutron-like output on the n-y discriminator. The upper edge of the window is set just above the highest incident neutron energy of interest, while the lower edge is set to limit the backsrownd. The energy signal and flight time are stored in a ewodimensional array in the PDP-15 computer. Oa read-out the low-energy threshold can be chosen to optimize peak-to-background ratios.

As indicated in sect. 3, the method of gating the team requires single-turn extraction from the cyclotron. A drif in any of the cyclotron parameters, or for example a momentary small arc from dee to ground, can shin the turn number of the extracted beam. To obverve and correct for this shift, the beam on target is monitored with a small plastic scintillator. The timeof titight signal of the prompt gammas from the target is displayed on an oscilloscope in the control room. The ratio of the number of pulses from the desired turn to the number from adjacent turns can then be observed and the cyclotron adjusted to maximize the ratio. By recording the time-of-light signal from the monitor in a muhichannel analyzer, the ratio is measured so that the integrated charge through the target can be comected. Typically, the correction is less than $1 \%$.

While the eflect of improper gating is small, it cin be important when strong contaminant peaks appea: in the neutron spectrum, since a "ghost" spectrum of the contaminant may overlay the primary spectrum from the reaction of interest.

The stop signal was obtained originally by inductively coupling a signal from the dee line. However, long-term drifts in the cyclotron parameters can result in wander in time of the beam pulses with respect to the cyclotron dee voltage. More recently the stop signal has been generated by capacitive pick-up from the beam pulses just upstream of the target. The principle is well known and has been in use for many years. The factors affecting the signal-to-noise ratio and rise time of the pulse, hence accuracy of timing, are discussed in a separate short technical note ${ }^{11}$ ).

The long-term time stability using the capacitive pick-up is of the order of $0.5 \mathrm{~ns}$ better than that obtained with the rf pick-off.

\section{Results}

Three spectra obtained for neutron-producing reactions are reproduced here to illustrate the properties of the system. The spectra of ${ }^{13} \mathrm{~N}$ and ${ }^{17} \mathrm{~F}$ obtained from the $(d, n)$ reaction on carbon and oxygen at $E_{s}=11.04 \mathrm{MeV}$ using as tar' ts a $40 \mu \mathrm{g} / \mathrm{cm}^{2}$. carbon foil and a $0.00015^{\prime \prime}$ Mylar foil $\left(\mathrm{C}_{10} \mathrm{H}_{8} \mathrm{O}_{4}\right)$ are shown in fig. 8. Note that the prompt $\gamma$-ray peak is considerably suppressed by $n-\gamma$ discrimination, and that the gating efficiency is essentially $100 \%$. The spectra were taken before the shielding was complete and before the addition of slits NS1 and NS2. This accounts for the large background, which has not been subtracted. The 
spectrum of ${ }^{14} \mathrm{O}$ from the reaction ${ }^{12} \mathrm{C}\left({ }^{3} \mathrm{He}, \mathrm{n}\right){ }^{14} \mathrm{O}$ at $E_{\mathrm{H}_{\mathrm{HC}}}=25.49 \mathrm{MeV}$ on a $80 \mu \mathrm{g} / \mathrm{cm}^{2}$ target of carbon is shown in fig. 9.

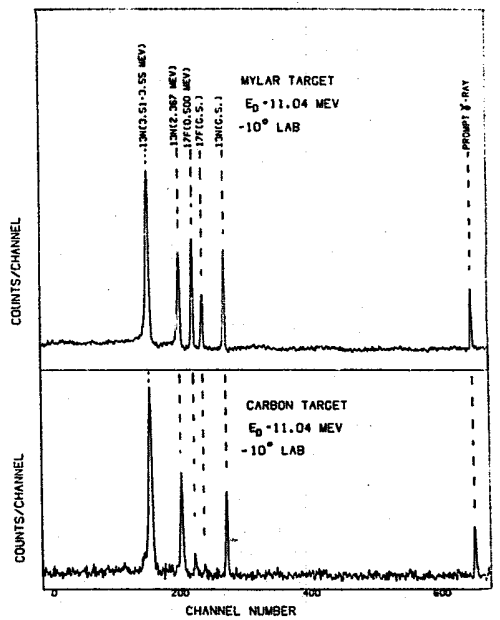

Fig. 8. Spectra of ${ }^{13} \mathrm{~N}$ and ${ }^{17} \mathrm{~F}$ ottained from the $(d, n)$ reaction on targets of carbon and Mylar.
These data were taken with a flight path of $37.5 \mathrm{~m}$ and the beam gated at $f / 8$ for the $(\mathrm{d}, \mathrm{n})$ reactions and $f / 9$ for the $\left({ }^{3} \mathrm{He}, \mathrm{n}\right)$ reaction. The width of the beam pulses on the target was typically $1.2 \mathrm{~ns}$ fwhm. For the $\left({ }^{3} \mathrm{He}, \mathrm{n}\right)$ data, singly ionized ${ }^{3} \mathrm{He}^{+}$was accelerated to $25.49 \mathrm{MeV}$, extracted, and stripped to ${ }^{3} \mathrm{He}^{+*}$ by sending the beam through a $20 \mu \mathrm{g} / \mathrm{cm}^{2}$ carbon foil placed at the first focus (FI) in the low-resolution beam line. The particle current available at the target using this method is typically $0.2 \mu \mathrm{A}$ for a pulsc width of $1.2 \mathrm{~ns}$ fwhm and a gating frequency $/ / 7$. This is som parable to the current obtained with deuterons and a factor of ten larger than for ${ }^{3} \mathrm{He}^{++}$. The disadvantases of accelerating ${ }^{3} \mathrm{He}^{+}$as compared to ${ }^{3} \mathrm{He}^{+}$are the more stringent stability requirements placed on the cyclotron. The ions must make twice the number of turns to extraction. The increase in time spread of the stripped beam, if any, was too small to be measured.

A number of improvements to the instrumeat can be made. It is plansed to improve the resolution by decreasing the diameter of each detector and increasing the total number in the array. Some improvement in the beam bunching without loss in intensity can be made, but only by improving the yield from the ion source. Unfortunately, it is the relatively low $n$ frequency inherent in the design of this cyclotron that results in the relatively large time spread for a given phase width. A significant reduction can be made in the background. That due to cosmic rays can be escentially eliminated, and the geometry of the shieiding and

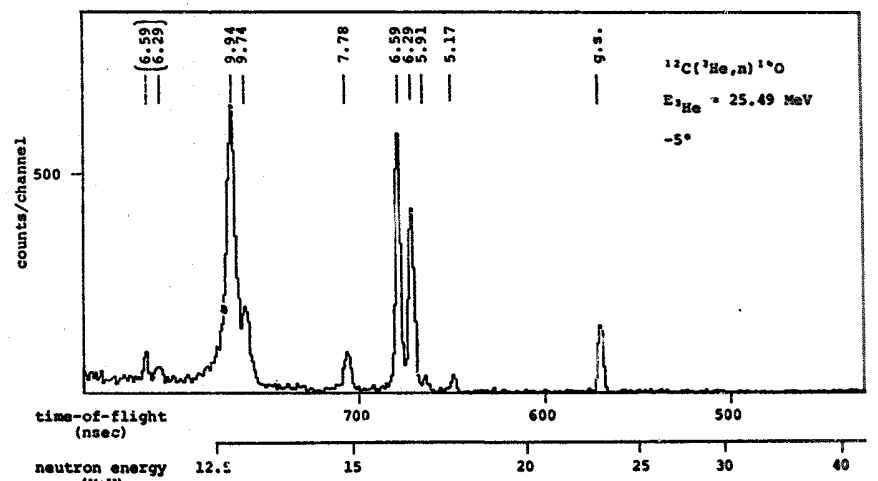

(nev)

Fig. 9. The spectrum of ${ }^{14} \mathrm{O}$ from the reaction ${ }^{12} \mathrm{C}\left({ }^{3} \mathrm{He}, \mathrm{n}\right)^{14} \mathrm{O}$ at $E_{\mathrm{lie}}=25.49 \mathrm{MeV}$. 
anti-scattering slits can be improved. The out-scattering of neutrons over the long flight path in air is appreciable, and more important, it fluctuates with energy. This can be improved by the use of helium-filled bags on by evacuation of the fight path.

It is a pleasure to acknowledge the contributions of J. Bardwick in the design of the new beam line. The willing and skilled assistance of $W$. E. Downer and other members of the teclnical staff of the Cyclotron laboratory is most appreciated.

\section{References}

1) A review of time of flight techniques is given by J. H. Neiler and W. M. Good in Fast neutron physics (Eds. Marion and Fonler: Interscience Publishers, Inc., New York, 1960) p. 509 .
2) R. Grismore and W. C. Parkinson, Rev. Sci. Instr. 28 (1957) 245.

3) G. F. Knoll, J. S. King and W. C. Parkinson, Phys. Rev. 131 (1963) 331.

4) R. H. Day and W. C. Parkinson, Nucl. Instr. and Meth. 111 (1973) 199.

5) To be submitted to Nucl. Instr. and Meth.

6) A. Papineau, P. Benezech and R. Maillard, J. Phys. Radium 21 (1960) 410.

7) W. C. Parkinson and J. Bardwick, Nucl. Instr. and Meth. 78 (1970) 24.5.

8) P. F. Julien, R. M. Polichar and W. C. Parkinson, Bull. Am. Phys. Soc. 18 (1973) 617.

9) P. F. Julien, Ph.D. Thesis (University of Michigan, Dec. 1973) to be published.

10) D. A. Gedeke and W. J. McDonald, Nucl. Instr. and Meth. $56(1967) 148$.

11) W. C. Parkinson, J. F. Petersen and D. C. DuPlantis, Nucl. Instr. and Meth. 117 (1974) 605. 